\section{Development of plasmids for quantitative detection of integrated lentiviral vectors and evaluation of culture time to perform vector titer by real-time quantitative polymerase chain reaction assay}

\author{
Elena Baiamonte, Mariella Bagliesi, \\ Valentina Motta, Barbara Spina, \\ Alice Pecoraro
}

UOC Ematologia per le Malattie Rare del Sangue e degli Organi Ematopoietici, Dipartimento di Oncologia ed Ematologia, AO Ospedali Riuniti Villa Sofia-Cervello, Palermo, Italy

\begin{abstract}
The accurate assessment of provirus copy number per cell (VCN/cell) is a fundamental issue in transgenesis as well as in gene therapy studies based on stably integrated vectors. To this end, real-time quantitative polymerase chain reaction (qPCR) is a powerful method but it is sensible to differences in quality or concentration of the two-plasmid preparations used for the construction of the standard curves. In order to minimize technical errors we included genome specific sequences (mouse or human) and vector specific sequences in the same plasmid. We evaluated the specificity and sensitivity of these bivalent plasmids by qPCR analysis on mouse and human genomic DNA containing a known number of a reporter lentiviral vector and we found that the system is reliable to measure up to $0.1 \mathrm{VCN} /$ cell. Here we have applied this assay to measure vector titer of virus stock preparations and to determine the optimal cell passages at which viral titration effectively reflects the number of integrated vectors.
\end{abstract}

\section{Introduction}

Transgenesis studies have represented the milestone to understand the molecular bases of cellular functions. Ex vivo genetic correction of autologous cells is an application of transgenesis in clinical setting, and in the last decade has represented an effective challenge to cure diseases otherwise incurable. Stable genetic modification of hematopoietic stem cells (HSC), mostly achieved with retroviral vectors (RV) [either $\gamma$-retroviral, $\gamma$-RV or lentiviral vector (LV)] is one example. This class of vectors integrated into the genomic DNA provides a permanent gene modification of the target cells and their abundant progeny, and consequently a long-term therapeutic effect. To date, HSC gene therapy mediated by RV has been successfully employed in nearly 100 patients affected by immune system or metabolic disorders and other monogenic diseases including $\beta$-thalassemia. ${ }^{1}$ Gene transfer efficiency (percentage of genetically modified cells) and vector copy number integrated per cell (VCN/cell) are factors that contribute to the successful outcome of the therapy in terms of efficacy and safety. Thus, an accurate measure of the transduction efficiency and of the relative number of vectors per cell is mandatory in preclinical and in clinical studies; in the latter, both during the quality control performed before the infusion of modified cells into the patient and after treatment to follow the gene transfer over time. For this reason, several research groups have developed in vitro assays to evaluate gene transfer, and real-time quantitative polymerase chain reaction (qPCR) is the most powerful tool especially in presence of very small amount of template. ${ }^{2}$ Moreover this fluorescence-based PCR method to detect nucleic acids is highly sensitive, specific and relatively easy to perform in automated machineries. The accuracy of quantification using this fluorescent based method greatly depends on the construction of standard curves prepared by a number of serial dilutions of two plasmids: one containing vector specific sequences, and the other containing cell host genome specific sequences as control. Minimal differences in the quality of these plasmid preparations or in the quantization of the plasmid concentrations may strongly affect the result. In order to minimize technical errors we constructed recombinant plasmids that include both sequences: vector specific sequences derived from the viral GAG sequence, and host cell specific sequences. Here we report that real time qPCR, using these plasmids, is highly specific and sensible to detect the VCN/cell of a GFP recombinant LVGFP. We have applied this assay to measure vector titer of virus stock preparations and to determine the optimal cell passages for a correct evaluation of integrated lentiviral vectors.

\section{Materials and Methods}

\section{Plasmid constructs}

To generate the mouse specific plasmid first we cloned a 1665 bp PCR fragment of mouse albumin in the pGEM-Teasy (Promega) MCS (primers FW: 5'TGCCTCAGCCTCCCAAGTA-3' REV: 5'-GGTCTCCTTATCGTCAGCCT-3') obtaining the intermedia pGEM-Teasy-mALB. Then a 2122 bp PstI-NdeI GAG
Correspondence: Alice Pecoraro, UOC Ematologia per le Malattie Rare del Sangue e degli Organi Ematopoietici, A0 Ospedali Riuniti Villa SofiaCervello, via Trabucco 180, 90146 Palermo, Italy. Tel.: +39.091.6802744 - Fax: +39.091.6880828. E-mail: a.pecoraro@ospedaliriunitipalermo.it

Key words: vector copy number, real-time quantitative polymerase chain reaction, lentiviral vector titer.

Acknowledgments: this work was partially supported by Fondazione Franco e Piera Cutino and by Assessorato Regionale delle Attività Produttive Sicilia - Regione Sicilia, LR23 Project RIMEDRI.

Contributions: all authors have contributed significantly to the research and declare no potential conflict of interests. EB performed the experiments, collected data and wrote the paper; $\mathrm{MB}$, VM, BS performed the laboratory work; AP coordinated the study, performed the experiments and wrote the paper.

Received for publication: 9 December 2013.

Revision received: 17 February 2014.

Accepted for publication: 13 March 2014.

This work is licensed under a Creative Commons Attribution 3.0 License (by-nc 3.0).

(C) Copyright E. Baiamonte et al., 2014

Licensee PAGEPress, Italy

Thalassemia Reports 2014; 4:2189

doi:10.4081/thal.2014.2189

restriction fragment obtained of pRLL SIN cPPT PGK eGFP WPRE LTR (LV-GFP) vector was cloned in the MCS of pGEM-Teasy-mALB obtaining the pGEM-Teasy-mALB-GAG. The same procedure was used to generate the human specific plasmid pGEM- Teasy-hALB-GAG; in this case a 1051 bp PCR fragment of human albumin was cloned (primers FW: 5'-CAGAAGGAAGTAATGTGTGT-3' REV: 5'-TGTGATAGCAATAGGGAGTT-3'). The two fragments, GAG and albumin were inserted in a way that the two regions complementary to the fluorescent probes were positioned at a distance of 2 $\mathrm{Kb}$ one to each other.

\section{Cell lines}

The murine erythroleukemia cell line MEL58546, the human HeLa cell line and the human $293 \mathrm{~T}$ cell line were grown at $37^{\circ} \mathrm{C}$ in the presence of $5 \% \mathrm{CO}_{2}$, in Dulbecco's modified Eagle's medium supplemented with $10 \%$ heat-inactivated FBS, $2 \mathrm{mmol} / \mathrm{L}$ l-glutamine, $0.1 \mathrm{mmol} / \mathrm{L}$ nonessential amino acids and the antibiotics (penicillin/streptomycin $10 \mathrm{~g} / \mathrm{mL}$ ) (all materials supplied by Gibco-Invitrogen, Life Science Technology, Grand Island, NY, USA).

\section{Generation of MEL and HeLa cells} containing a known vector copy number A total of $5 \times 10^{5}$ MEL and HeLa cells were 
transduced with $\mathrm{LV}$-GFP vector at a modest MOI to limit the VCN/cell as reported elsewhere. ${ }^{3}$ Sixteen hours after transduction the cells were washed and plated at one cell per well in 96-well flat-bottomed dishes. Individual clones were expanded and screened for the presence (GFP fluorescent cells) and for the integrated vectors by Southern blot analysis using specific radiolabeled probes.

\section{Vector production and titration via GFP expression and via quantitative polymerase chain reaction}

Viral stocks were generated by co-transfection into 293T cells of the gene transfer plasmid (LVGFP) with the envelope plasmid (VSV-G) and the packaging plasmids (pMDLg/pRRE and pRSVREV). Vector production was performed as reported elsewere. ${ }^{4}$ Viral titers were determined by transduction of HeLa cells with serial dilution of the vector stock, and GFP expression was measured by flow cytometer (FC-500; Beckman Coulter, Inc., Brea, CA, USA) at day 5 of culture. The titer was calculated using the following formula: number of cells $\times$ dilution factor $\times$ percentage of GFP positive cells. To measure vector titers by real time qPCR an aliquot of cells after transduction was expanded and splitted every two days and genomic DNA were prepared at 8, 12, 14 and 16 days of culture that correspond to 2,3,4 and 5 cellular passages respectively. Virus titers were measured using the following formula: number of cells $\times$ dilution factor $\times$ VCN, using the primers described in the section below.

\section{Quantitative real-time polymerase chain reaction analysis}

Real-time quantitative PCR was performed in ABI PRISM 7900 sequence detection system (Applied Biosystems, Inc., Foster City, CA, USA) using the following primers and probes: GAG-Fwr: 5'-GGAGCTAGAACGATTCGCAGTTA-3', GAG-Rev: 5'GGTTGTAGCTGTCCCAGTATTTGTC-3', GAG probe: 5'-FAM-ACAGCCTTCTGATGTTTCTAACAGGCCAGG -TAMRA-3'; mALB-Fwr: 5'-GGAAAAGTGCTGCGCTGAAG-3', mALB-Rev: 5'-TTGGCTCATGGAAACCTACCA-3', mALB probe: 5'-VIC-AATCCTCCCGCATGCTACGGCA-TAMRA-3'; hALB-Fwr: 5'-TGAAACATACGTTCCCAAAGAGTTT-3', hALB-Rev: 5'-CTCCTTCTCAGAAAGTGTGCATAT-3', hALB probe: 5'VIC-TGCTGAAACATTCACCTTCCATGCAGATAMRA-3'. Each DNA sample was run in triplicate in $25 \mu \mathrm{L}$ reaction volume using Taqman Universal PCR Master Mix (Applied Biosystems). Thermal cycling was started for $10 \mathrm{~min}$ at $95^{\circ} \mathrm{C}$, followed by 40 thermal cycles of $15 \mathrm{~s}$ at $95^{\circ} \mathrm{C}$ and $1 \mathrm{~min}$ at $60^{\circ} \mathrm{C}$. Vector copy number/cell was calculated using the following formula: (quantity mean of GAG sequence/quantity mean of ALB sequence) $\times 2$ (or $\times 3$ ). 2 -fold factor was used in the case of DNA from the diploid cells (MEL); 3-fold factor was used in the case of DNA from the triploid cells (HeLa).

\section{Results and Discussion}

\section{Plasmid constructions}

We constructed a mouse specific plasmid (pGEM-Teasy-mALB-GAG) and a human specific plasmid (pGEM-Teasy-hALB-GAG) that carry the sequences from mouse or human albumin respectively, and the viral specific fragment derived from GAG sequences into pGEM-Teasy. We cloned the two fragments in a way that probe specific regions were positioned at a distance of $2 \mathrm{~Kb}$ one to each other.

\section{Standard curves setting}

Using pGEM-Teasy-mALB-GAG and pGEMTeasy-hALB-GAG we setted standard curves using a serial of log dilutions starting from a concentration of $10^{7}$ copies of plasmid/ $\mu \mathrm{L}$. The analysis of the amplification reactions showed that there was no quenching between the two fluorescent signals generated by the adjacent probes at the distance of $2 \mathrm{~Kb}$. In both mouse and human curves, the Ct (threshold Cycle) values interpolated a straight line with slope values of 3.33 and $r^{2}=0.99$. These values revealed an optimal standard curve construction.

\section{Quantization of the absolute number of integrated vector per cell}

We transduced MEL and HeLa with a LV-GFP vector and we identified cells bearing the vector by flow cytometry. We screened individual clones to determine the VCN cell by Southern blot analysis (data not shown). We used genomic DNA extracted from clones that resulted in $1,2,3$, and 4 copies/cell to perform real time qPCR assays using our bivalent plasmids. The results from several experiments using different plasmid preparations and dilutions always confirm the number

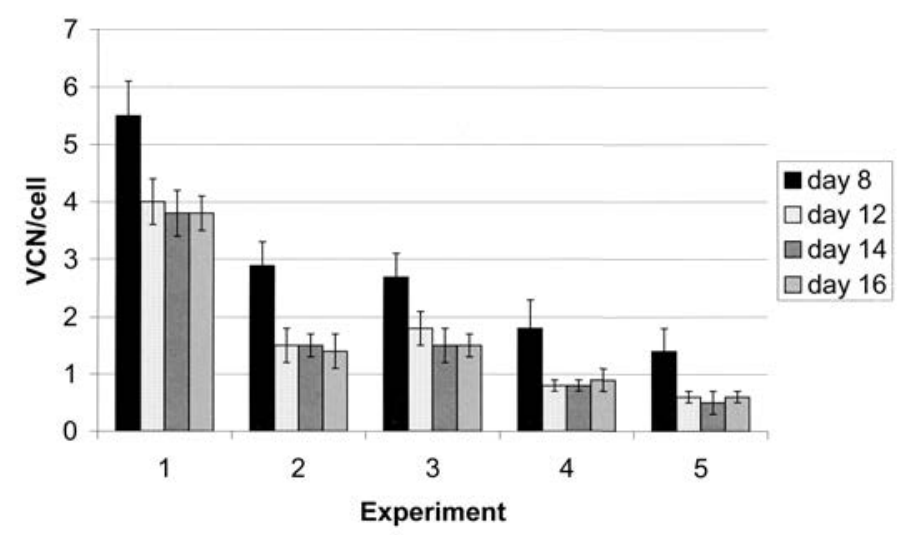

Figure 1. Vector copy number per cell (VCN/cell) analysis in HeLa cells at different time point after transduction with different viral stock preparations. VCN/cell are measured by quantitative polymerase chain reaction in DNA from HeLa cells at day 8, 12, 14 and 16 after transduction with the lentiviral vector-GFP vector. On the ordinate axis is reported the VCN/cell, on the abscissa are reported 5 different experiments (1 to 5). Day 8, 12, 14 , and 16 corresponds to $2,3,4$, and 5 cell passages respectively. 


\section{Conclusions}

One of the crucial aspects in transgenesis studies is to determine the exact amount of genetically modified cells and the number of transgenes per cell. Real-time qPCR is a usefulness assay to evaluate the quantity of vector integrated in the host genome also in the presence of a minimal amount of nucleic acids.

The correct quantification by this method depends on the construction of standard curves prepared by serial dilutions of two plasmids, one carrying vector specific sequences and the other carrying host cell genome specific sequences as control.

The correct setting of an absolute standard curve procedure requires several parameters including the DNA purity since RNA contamination may inflate DNA concentration and ultimately the dilutions necessary to prepare a correct standard curve. Difference in the concentrations of the two plasmids necessary to prepare the standard curves is directly reflected in quantification errors by qPCR assay.

In order to limit technical errors we inserted the aforementioned sequences, vector specific sequences (GAG region) and host cell genome specific sequences, in the same plasmid. In particular we generated a mouse specific plasmid and a human specific plasmid. Here we showed that there was no quenching between the two probes (GAG and ALB) when the two sequences are positioned at $2 \mathrm{~Kb}$ of distance one to each other. The VCN performed by real time qPCR using these plasmids was validated by Souther blot analysis and was highly specific and sensitive (up to $0.1 \mathrm{VCN} / \mathrm{cell}$ ). Here we used this method for the titration of LV-GFP vector preparations and observed that titer values obtained by qPCR were 3 times higher than those obtained by measuring the frequency of GFP expressing cells.

We also determined that 12 days of culture (or 3 cell passages) after transduction are necessary to determine the correct number of integrated lentiviral vectors per cell. Before this time the values of VCN/cell result overextimated due to the presence of not integrated DNA circles that are subsequently lost during the cell divisions. In conclusion the bivalent plasmids we constructed are a useful tool to minimize technical errors that can occur in qPCR.

\section{References}

1. Rivière I, Dunbar CE, Sadelain M. Haematopoietic stem cell engineering at a crossroads. Blood 2012;119:1107-16.

2. Delenda C, Gaillard C. Real-time quantitative PCR for the design of lentiviral vector analytical assay Gene Ther 2005;12:36-50.

3. D'Apolito D, Baiamonte E, Bagliesi M, Di Marzo R. The sea urchin sns5 insulator protects retroviral vectors from chromosomal position effects by maintaining active cromatine structure. Molecular Ther 2009;17:1434-41.

4. Lotti F, Menguzzato E, Rossi C, et al Transcriptional targeting of lentiviral vectors by long terminal repeat enhancer replacement. J Virol 2002;76:3996-4007.

5. Li L, Olvera JM, Yoder KE, et al. Rule of the non-homolougous DNA end joining pathway in the early steps of retroviral infection. EMBO J 2001;20:3272-81. 